\title{
Aprovechamiento de la energía solar para el Área Académica de la Escuela de Aviación Policial mediante un sistema fotovoltaico con conexión a red
}

\author{
Harnessing solar energy for the Academic Area of the Police Aviation School \\ through a photovoltaic system with grid connection
}

\section{Aproveitamento da energia solar para a Área Acadêmica da Escola de Aviação Policial através de um sistema fotovoltaico com conexão de rede}

\author{
Giovanni Andrés Vargas G. | Santiago Alejandro Gil Baena | John Edisson Díaz Figueroa \\ | Luis Miguel Otálora Dueñas* \\ Policía Nacional de Colombia, San Sebastián de Mariquita, Colombia
}

- Fecha de recepción: 2017-06-29

- Fecha concepto de evaluación: 2019-09-06

- Fecha de aprobación: 2019-09-1I http://dx.doi.org// 0.22335/rlct.vI Ii2.446
Para citar este artículo / To reference this article / Para citar este artigo: Vargas G., G.A., Gil Baena, S.A., Díaz Figueroa,J.E., \& Otálora Dueñas, L. M. (2019).Aprovechamiento de la energía solar para el Área Académica de la Escuela de Aviación Policial mediante un sistema fotovoltaico con conexión a red. Revista Logos Ciencia \& Tecnología, II (2), 46-59. http://dx.doi.org//0.22335/rlct.vI Ii2.446

\section{RESUMEN}

El artículo presenta el diseño de un sistema fotovoltaico con conexión a red para el Área Académica en la Escuela de Aviación Policial (ESAVI) de la ciudad de Mariquita, Tolima. La investigación aplicada es del tipo preexperimental, alcance descriptivo y enfoque cuantitativo. Los resultados muestran un consumo energético para la ESAVI de $1.930,6 \mathrm{I} \mathrm{kWh} /$ día y $38.503,34 \mathrm{kWh} / \mathrm{mes}$ para diciembre del 2015; además, se propone un sistema fotovoltaico con conexión a red o generación distribuida (GD) con una capacidad de $63,4 \mathrm{kWh} /$ día y que equivale al $30 \%$ de energía consumida por la misma área, el cual está compuesto por 63 paneles solares de 260 watt, un inversor trifásico de capacidad 15.000 watt y un contador bidireccional clase 2. Asimismo, se calculó el valor actual neto con un tiempo de II años para la recuperación económica y la tasa interna de retorno para el 204 I de 10,70\%, que indican la viabilidad financiera para la implementación del sistema fotovoltaico diseñado. También se espera contribuir con la mitigación del cambio climático y, por ende, al cuidado del medioambiente con la no emisión de $0,39 \mathrm{tCO}_{2 \mathrm{eq}}$ por mes y la generación de cultura ambiental en los funcionarios y estudiantes de la institución.

Palabras clave: energía, fotovoltaico, panel solar, generación distribuida, Escuela de Aviación

\section{SUMMARY}

The article presents the design of a grid-connected photovoltaic system for the Academic Area at the Police Aviation School (ESAVI) in the city of Mariquita, Tolima. The applied research is of the pre-experimental type, with a descriptive scope and quantitative approach. The results show energy 
consumption for ESAVI of I,930.6I kWh/day and 38,503.34 kWh/month by December 20I5. In addition, a photovoltaic system is proposed with connection to grid or distributed generation (GD) with a capacity of $63.4 \mathrm{kWh} /$ day and that is equivalent to $30 \%$ of energy consumed by the same area. This is composed of sixty-three 260-watt solar panels, a three-phase inverter of 15,000-watt capacity, and a bidirectional meter class 2 . Likewise, the net present value was calculated with a time of II years for the economic recovery and the internal rate of return for $204 \mathrm{I}$ of $10.70 \%$, which indicate the financial viability for the implementation of the designed photovoltaic system. It is also expected to contribute to the mitigation of climate change and, therefore, to caring for the environment with the emission of 0.39 tCO2eq per month and the development of environmentally aware culture in the officials and students at the institution.

Keywords: Energy, photovoltaic, solar panel, distributed generation, Aviation School

\section{SUMÁRIO}

O artigo apresenta o projeto de um sistema fotovoltaico com conexão de rede para a Área Acadêmica da Escola de Aviação Policial (ESAVI) da cidade de Mariquita, Tolima. A pesquisa aplicada é do tipo pré-experimental, com escopo descritivo e abordagem quantitativa. Os resultados mostram um consumo de energia para ESAVI de 1.930,61 kWh/dia e 38.503,34 kWh/mês em dezembro de 2015; além disso, propõe-se um sistema fotovoltaico com conexão de rede ou geração distribuída (GD) com capacidade de $63,4 \mathrm{kWh} /$ dia e equivalente a $30 \%$ da energia consumida na mesma área, o qual está composto por 63 painéis solares de 260 watts, um inversor trifásico com capacidade de 15.000 watts e um medidor bidirecional classe 2. Da mesma forma, o valor presente líquido foi calculado com um tempo de II anos para a recuperação econômica e a taxa interna de retorno para 204 I de $10,70 \%$, que indicam a viabilidade financeira para a implementação do sistema fotovoltaico projetado. Espera-se também contribuir para a mitigação da mudança climática e, portanto, para o cuidado do meio ambiente com a não emissão de $0,39 \mathrm{tCO}$ eq por mês e a geração de cultura ambiental nos funcionários e alunos da instituição.

Palavras-chave: Energia, fotovoltaico, painel solar, geração distribuída, Escuela de Aviación

El actual cambio climático como consecuencia del incremento de los gases de efecto invernadero, sugiere que es tiempo de cambiar la forma como se consume y se produce la electricidad. Además, la crisis energética es uno de los problemas más importantes a los que nos enfrentamos en los últimos años. Las fuentes de energía renovable recientes desempeñan un papel primordial para hacer frente a estos problemas. El sistema de generación fotovoltaica se ha incrementado de manera rápida en las últimas décadas, y según el más reciente informe sobre la energía fotovoltaica instalada, la meta de producción de I00GW se alcanzó a finales del 2012, y la mayoría de estos están conectados a la red (Islam, Mekhilef \& Hasan, 2015; Tsang \& Chan, 2014).

La Policía Nacional de Colombia no es indiferente a esta realidad, tiene la voluntad de desarrollar e implementar tecnologías que permitan cada día mejorar los procesos de calidad al interior de la misma. Por esta razón, se es- tán generando propuestas encaminadas a la reducción de la contaminación y el aprovechamiento y uso moderado de los recursos económicos, sociales, ambientales, entre otros.

Además, la utilización de un sistema fotovoltaico en el Área Académica de la Escuela de Aviación Policial (ESAVI), permitirá no solo reducir el consumo de energía eléctrica en cada aula de clase y en las oficinas ubicadas en este sector, sino también cuidar el medioambiente. Asimismo, esta tecnología emplea una fuente de energía limpia y gratuita, su operación es automática y silenciosa, requiere poco mantenimiento y es amigable con el ambiente (Domínguez-González, 2012).

La metodología que se utilizará para este proyecto será la generación distribuida (GD) o conexión a red;el cual propone reducir el consumo de energía proveniente del sistema interconectado de la ESAVI, con la implementación de una 
red de energía solar fotovoltaica. El proyecto se realizará en tres fases: en la primera, se caracterizará el sistema de baja tensión de la red de suministro de energía a través de mediciones con analizadores de potencia que permite hacer monitoreos continuos, con el fin de establecer la calidad de la energía y las fluctuaciones de carga de la red.

En la segunda fase, se hace la caracterización de la producción de energía fotovoltaica, dimensionando el sistema de paneles solares y sus componentes; además, su respectivo montaje y las conexiones a la red eléctrica de la Escuela.

En la tercera fase, se incluyen los costos de los diferentes componentes del sistema, se ingresa la información obtenida de las redes de las empresas del sector eléctrico, se realiza un estudio técnico-económico del proyecto; el cual permitirá establecer los costos de la instalación y la dimensión del sistema, se establecen las ventajas económicas de la instalación, los retornos de inversión y la reducción de gases de efecto invernadero.

\section{Método}

El estudio es preexperimental, con un alcance descriptivo y un enfoque cuantitativo, puesto que analiza como principal objeto de estudio el diseño de un sistema fotovoltaico con conexión a red para la ESAVI; ello, teniendo en cuenta para la caracterización de la energía eléctrica, la metodología de gestión total eficiente de la energía (Campos-Avella, Gómez-Dorta \& Santos-Macías, 1997) y para el diseño del sistema fotovoltaico, la generación distribuida (GD), y como indicadores se usaron la línea base de consumo energético por hora y día de la semana, la potencia instalada por área y la hora solar estándar (HSS).

\section{Caracterización energética del}

\section{Área Académica de la ESAVI}

Para realizar la caracterización energética en el Área Académica de la Escuela de Aviación Policial, se llevó a cabo la línea base de consumo energético; además, se determinó la capacidad instalada, la estratificación de aulas u oficinas y equipos con los diagramas de Pareto, teniendo en cuenta el consumo energético.

Línea base de consumo energético del Área

Académica de la Escuela de Aviación Policial

Para establecer la línea base de consumo energético fue indispensable determinar las variables de energía eléctrica por hora, utilizando un analizador de potencia para establecer los registros de voltaje.

\section{Variables de energía eléctrica por hora}

Para hacer la toma de datos de las variables de la energía eléctrica en el Área Académica, se instaló en el transformador ubicado en esta área, el analizador de potencia marca Hioki 3360-2I; el cual se instaló, de acuerdo con la norma de calidad de energía NTC 500I (Norma Técnica Colombiana [NTC], 2008), en el interruptor automático principal (véase figura I), desde el 4 de diciembre hasta el II del mismo mes del 20I5, y el tratamiento de los datos se hizo con el software SFI00I que viene con el equipo; posteriormente, se descargaron en archivos CSV y se realizó su tratamiento estadístico descriptivo en Excel por hora del día.

Figura I. Analizador de potencia Hioki 3360-2I en el transformador del Área Académica

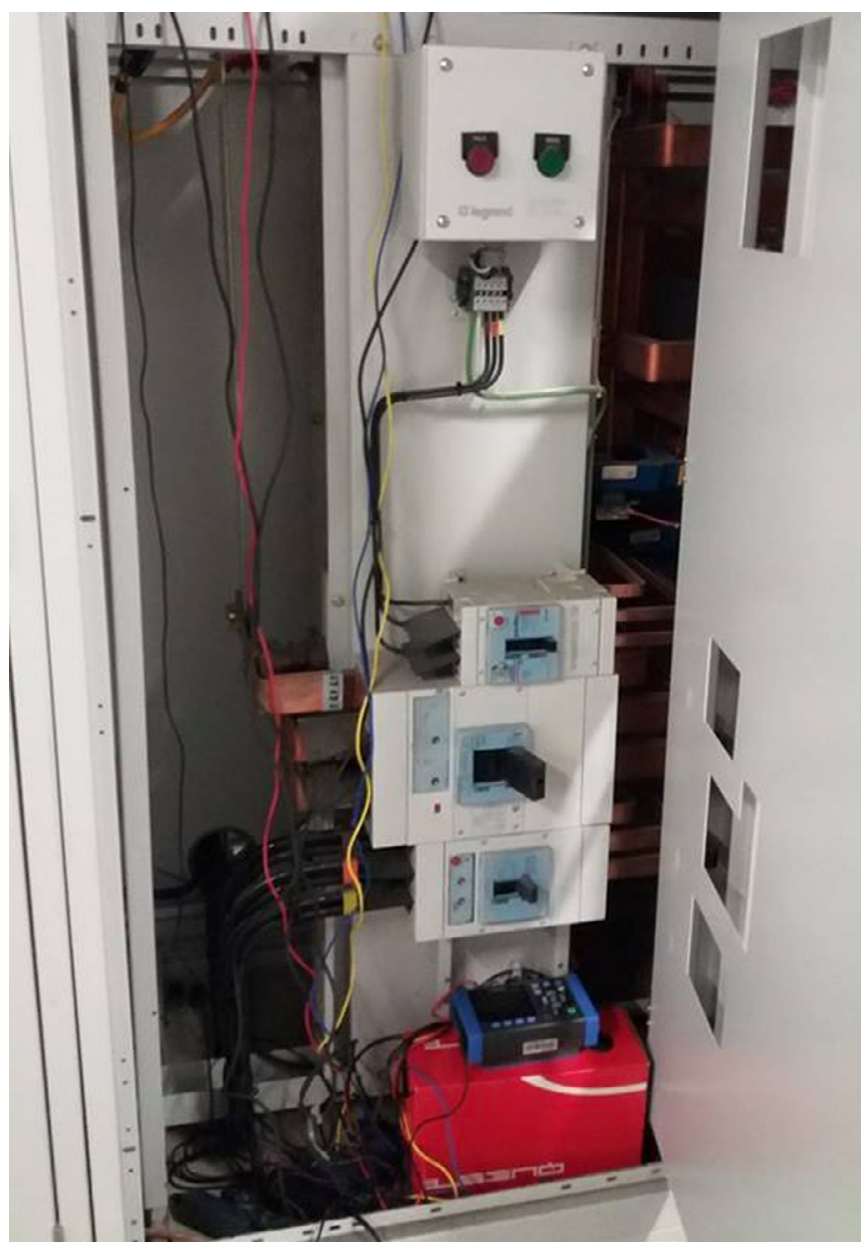

Teniendo en cuenta la hora promedio por semana, se hicieron las siguientes gráficas: 
Figura 2. Consumo de energía activa (kWh) del Área Académica de la Escuela de Aviación Policial

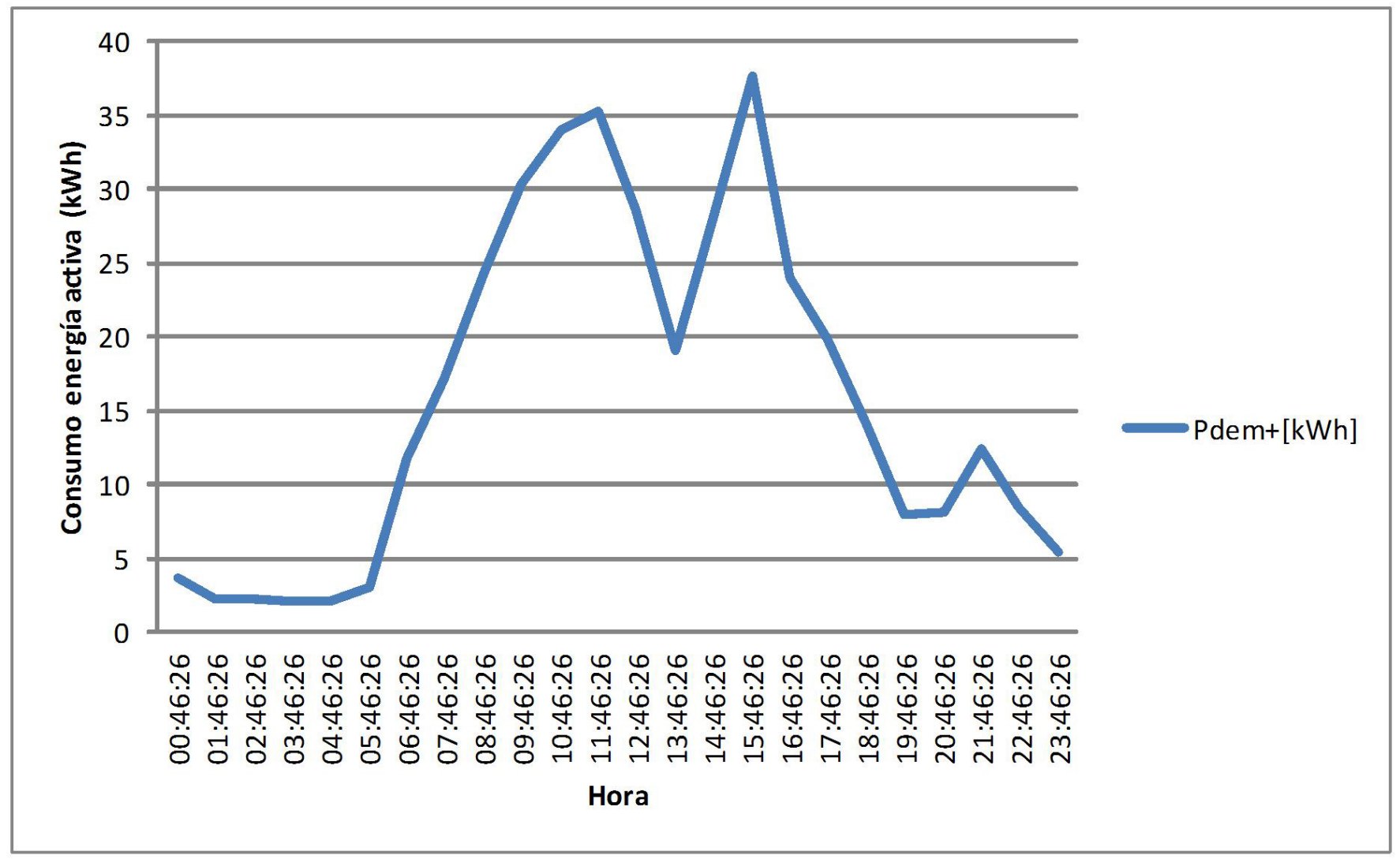

Fuente: elaboración propia a partir de los datos del software SF00I.Año 2015.

a) Energía activa (kWh). En la figura 2 se evidencia que el mayor consumo promedio de energía activa es de $23,38 \mathrm{kWh}$. Este se presenta entre las 6 a.m. y las 6 p.m., que es el horario normal de clase de los estudiantes de la Escuela de Aviación Policial. Sin embargo, debe tenerse en cuenta que hay consumos de energía activa elevados entre las 12 de la noche y las 6 a.m. con un valor de 2,48 kWh, y de las 6 p.m. a las 12 de la noche con un valor de $9,43 \mathrm{kWh}$, que no es el horario normal de trabajo de la escuela y que se debe, posiblemente, al encendido de las luminarias de los pasillos, equipos encendidos, entre otras posibles actividades.

De igual forma, se evidencia que en la franja de $12 \mathrm{~m}$. a 2 p.m. disminuye el consumo energético, puesto que las personas tienen la hora de almuerzo; sin embargo, el consumo energético aún es alto con un valor de $27,68 \mathrm{kWh}$; lo cual indica que se dejan equipos encendidos como aires acondicionados, luminarias, entre otros, sin haber personas dentro de las aulas académicas. b) Energía reactiva (kVArh). En la figura 3 se identifica que la mayor generación promedio de energía reactiva es de 3,64 kVArh y se presenta entre las 6 a.m.y las 6 p.m., que es el horario normal de clase de los estudiantes de la Escuela de Aviación Policial; este se puede dar por el uso de aires acondicionados.

Sin embargo, debe tenerse en cuenta que hay generación de energía reactiva elevada entre las 12 de la noche y las 6 a.m. con un valor de 0,90 kVArh, y de las 6 p.m. a las 12 de la noche con un valor de I,89 kVArh, que no es el horario normal de trabajo de la escuela. Este elevado registro se puede dar por la utilización de aires acondicionados cuando se trabaja hasta altas horas de la noche y madrugada.

De igual forma, se evidencia que en la franja de $12 \mathrm{~m}$. a 2 p.m. disminuye la generación de energía reactiva, puesto que las personas tienen la hora de almuerzo; sin embargo, se detecta que la energía reactiva aún es alta con un valor de 4,46 kVArh; lo cual indica que se dejan equipos 
Figura 3. Consumo de energía reactiva (kVArh) del Área Académica

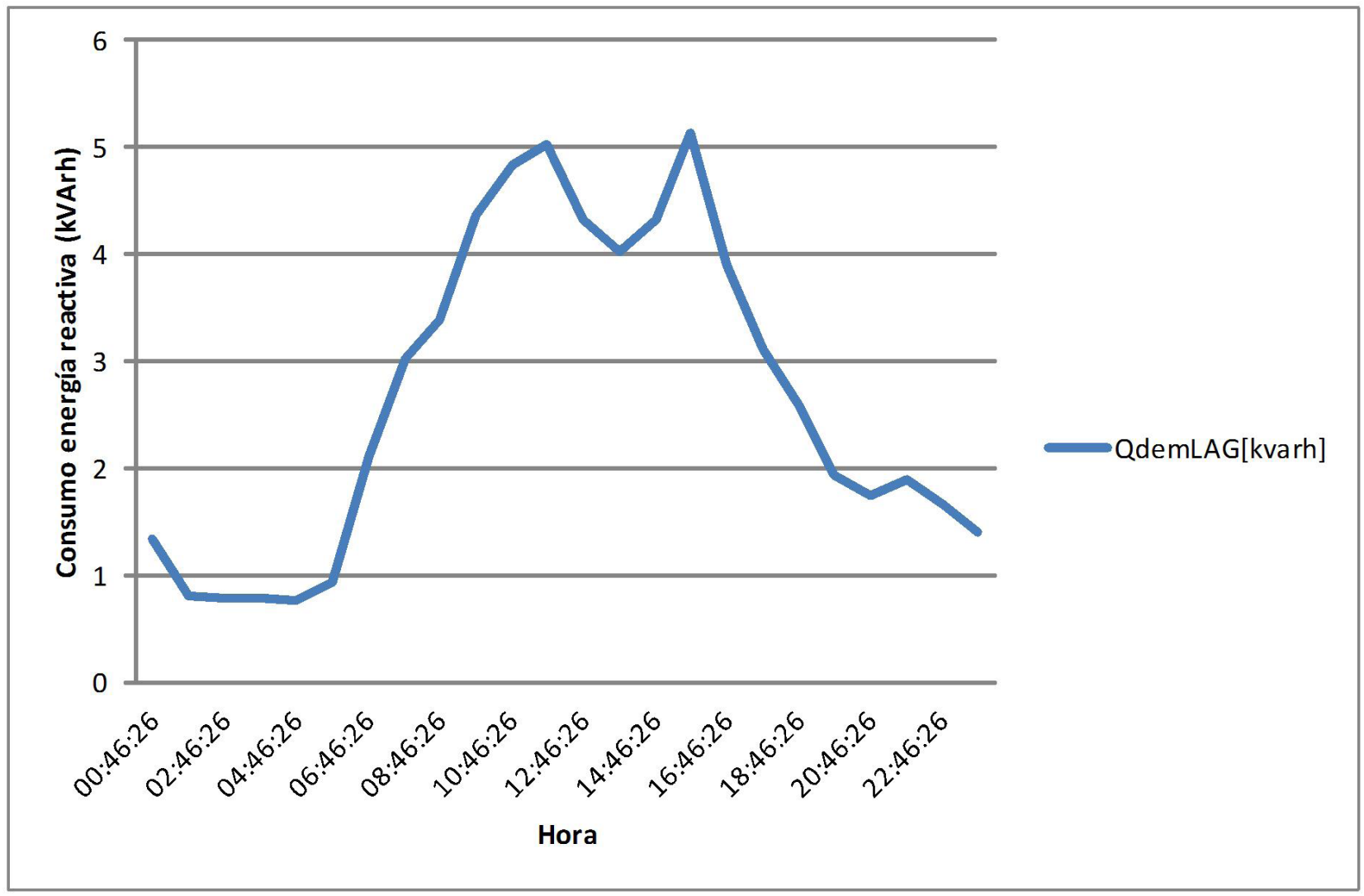

Fuente: elaboración propia a partir de los datos del software SF00I.Año 2015.

encendidos como los aires acondicionados o motores eléctricos.

Perfil de consumo de energía activa

$(\mathrm{kWh})$ por día de la semana

En la figura 4 se evidencia que el mayor consumo promedio de energía activa se encuentra entre 17,74 y 21,78 $\mathrm{kWh}$ y se presenta de lunes a viernes, siendo este el horario normal de clase de los estudiantes de la Escuela. Sin embargo, los fines de semana (sábado y domingo) se presenta un consumo promedio de energía activa de 6,96 $\mathrm{kWh}$, que es elevado, teniendo en cuenta que no hay programación de clases, las oficinas laboran y este consumo se puede dar por los equipos encendidos en el Área Académica, iluminación de pasillos, entre otros.

\section{Capacidad de potencia instalada del Área Académica}

La capacidad instalada se elaboró con base en la potencia nominal (W) y cantidad de equipos ubicados en cada zona del Área Académica. En la tabla I se muestra el registro.
Tabla I. Capacidad de potencia instalada (W) del Área Académica

\begin{tabular}{|c|c|}
\hline Área o aula académica & Capacidad instalada (W) \\
\hline $\mathrm{I}$ & 17.216 \\
\hline 2 & 17.537 \\
\hline 3 & 15.183 \\
\hline 4 (Sala de idiomas) & 21.852 \\
\hline 5 & 17.323 \\
\hline 6 & 16.360 \\
\hline 7 & 16.360 \\
\hline 8 & 17.430 \\
\hline 9 & 7.805 \\
\hline Biblioteca & 19.242 \\
\hline Oficinas & 43.607 \\
\hline Simuladores (avión y helicóptero) & 32.364 \\
\hline Baños & 544 \\
\hline Pasillo & 275 \\
\hline Hall mesas pin pon & 250 \\
\hline Total & 243.350 \\
\hline
\end{tabular}


Figura 4. Consumo de energía activa (kWh) por día de la semana

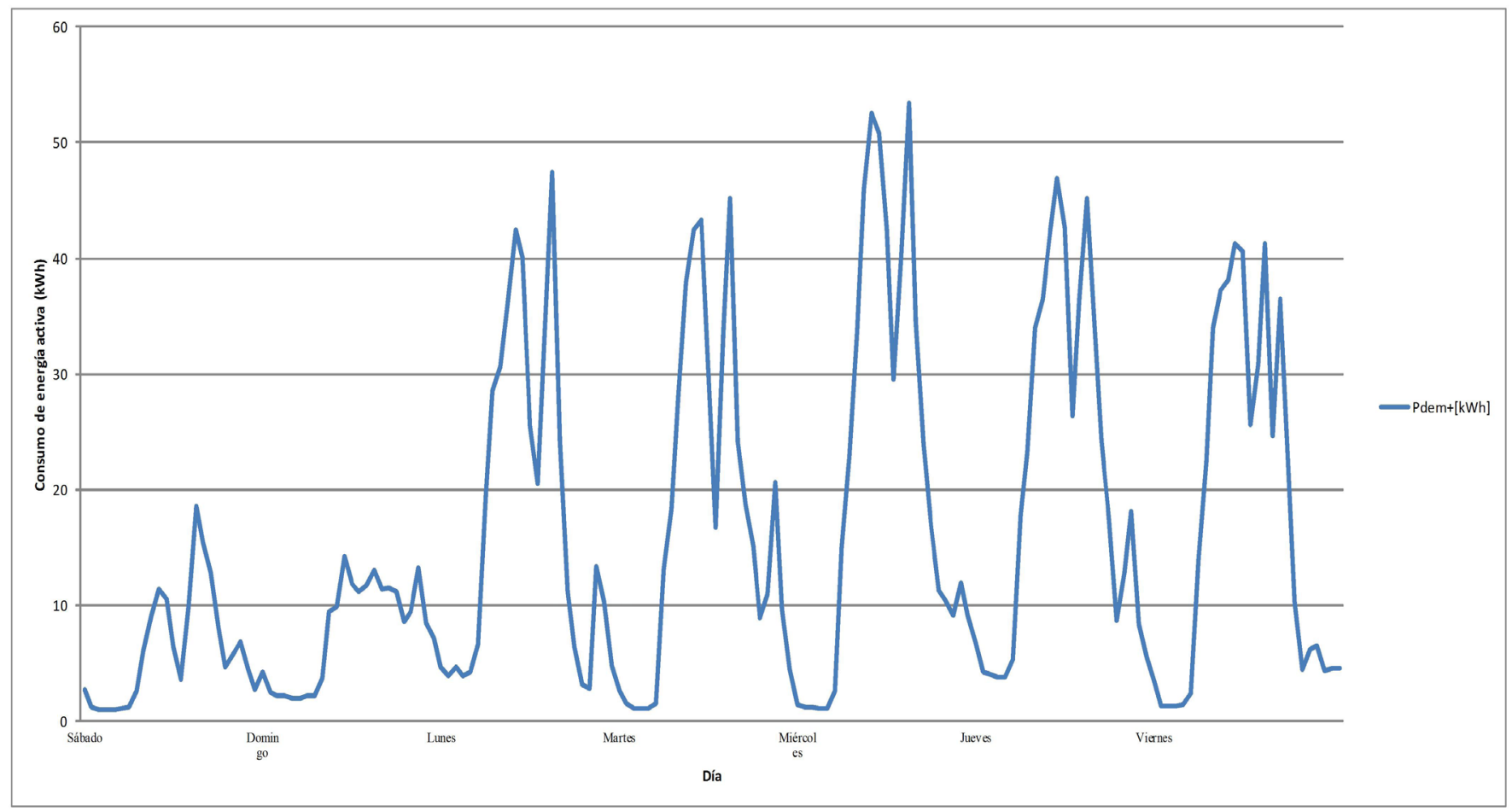

Fuente: elaboración propia a partir de los datos del software SF00I.Año 2015.

En el registro de la tabla I se observa que las áreas con mayor potencia instalada son oficinas con $43.607 \mathrm{~W}$, seguidas de los simuladores con $32.364 \mathrm{~W}$, aula 4 (centro de idiomas) con $21.852 \mathrm{~W}$ y la biblioteca con 19.242 W.

\section{Estratificación del Área Académica de la Escuela de}

Aviación Policial, por oficinas o aulas y equipos

La estratificación se realizó por subáreas establecidas para este estudio (aulas, oficinas, pasillos y hall), teniendo en cuenta la capacidad de potencia instalada y el funcionamiento de los equipos por hora, día, mes y año.

Se logró determinar que las áreas con mayor consumo energético $(\mathrm{kWh} / \mathrm{mes})$ son: oficinas con $10.181,85$, centro de idiomas con 3.446,97, los simuladores con 3.050,77, la biblioteca con $2.735,53$ y las aulas académicas del 2 al 8 con un consumo promedio de 2.700 .

De igual forma, para la estratificación por equipos se tomó solo las oficinas, puesto que los equipos en casi todas las áreas son los mismos; además, se determinó que el equipo más crítico, que cumple con la ley del Pareto $(20 \%$ de los equipos que consumen el $80 \%$ de la energía), es el aire acondicionado con un consumo de $8.445,75 \mathrm{kWh} /$ mes. En caso de realizarse un estudio de gestión energética al Área Académica, este sería el equipo en el que deben centrarse los diagnósticos mediante el cálculo de cargas térmicas, recambio por equipos de alta eficiencia o bioclimatizadores, desincrustantes o cambio de refrigerantes que permitan mejorar la eficiencia de estos aires.

\section{Diseño del sistema fotovoltaico con conexión a red o generación distribuida (GD)}

La metodología para el dimensionamiento de un sistema fotovoltaico como opción de generación distribuida en redes de baja tensión o interconectado a la red eléctrica se basó en dos casos de estudio para el 2012 en la ciudad de Bogotá. El primero se elaboró para la Universidad Nacional (Hernández-Mora, 20I2), y el segundo en la Universidad Distrital Francisco José de Caldas (Patiño-Abella, Tello-Reyes \& Hernández-Mora, 2013).

Por otro lado, también se realizó un cálculo con la metodología utilizada por Carneiro (2009) en la Universidad Do Minho, en Brasil, en el 2009, y arrojó como resultado el mismo dato de paneles de las metodologías anteriores, razón por la cual se decidió dejar las primeras teniendo 
en cuenta la facilidad para calcular y que se parte de la energía activa requerida ( $A C$ y $D C$ ).

La metodología que se trabajó estuvo de acuerdo con Vargas-Galván (20I5); el cual, basado en los anteriores estudios, logró establecer el modelo de cálculo que se muestra a continuación:

a) Dimensionamiento del sistema. Dado que la luz solar es un recurso cuya intensidad no es constante y se encuentra disponible solo en una franja horaria del día, el dimensionamiento de un sistema fotovoltaico se debe realizar a partir de la energía requerida por las cargas según la potencia consumida y la cantidad de horas de funcionamiento, puesto que un dimensionamiento a partir de la potencia supone un suministro de corriente continua.

La metodología aplicada para el dimensionamiento del sistema, en una primera instancia, consiste en la definición de la energía total a suministrar; es decir, se establece la potencia nominal de las cargas y la cantidad de horas del día en las que se encuentra funcionando.

- La energía que demandan las cargas de DC y AC se determinan de la siguiente forma - véanse ecuaciones (I) y (2)-:

Energía $_{D C}=\sum_{i}^{k} P_{i} * n_{i}[\mathrm{Wh}]$

Donde $i=$ elemento considerado, $P i=$ potencia nominal del elemento, $n i=$ número de horas al día que se encuentra en uso.

Energía $_{A C}=\frac{\sum_{i}^{k} P_{i} *_{i}}{F I}[W h]$

Donde $i=$ elemento considerado, $\mathrm{Pi}=$ potencia nominal del elemento, $n i=$ número de horas al día que se encuentra en uso, $F I=$ factor de inversión.

En el caso de disponer de cargas de AC, se necesita un inversor para la alimentación de dichas cargas, por lo que el factor de inversión está asociado a la eficiencia de operación de dicho inversor.A continuación, se define la energía que debe suministrar el sistema para atender la demanda de las cargas dispuestas para el sistema - véanse ecuaciones (3) y (4)-.

$$
\begin{aligned}
& \text { Energía }_{\text {Total }}=\text { Energía }_{A C}+\text { Energía }_{D C}[\mathrm{Wh}] \\
& \text { Energía }_{\text {Total_Corregida }}=\left(\text { Energía }_{\text {Total }}\right) *(I+F S)[W h]
\end{aligned}
$$

El término FS corresponde al factor de seguridad; el cual se dispone para realizar un sobredimensionamiento y en orden dar más confiabilidad al sistema y compensar cualquier tipo de pérdida de energía que se pueda presentar. Además, para establecer las condiciones de carga y corriente presentes en el sistema dispuesto, las siguientes variables se encuentran a fin de seleccionar los elementos adecuados que permitirán hacer la instalación del sistema. En el caso de este estudio, se tomó la energía alterna de la base de datos de capacidad instalada de la ESAVI que se había elaborado.

- Tamaño del generador fotovoltaico. En este apartado se evaluará la cantidad de módulos en paralelo y en serie que conformarán el arreglo dispuesto para hacer la alimentación del sistema.

Cantidad de módulos en serie. Los módulos fotovoltaicos se encuentran en tensiones típicas de $12 \mathrm{Vdc}$ o 24 Vdc, por lo que a partir del establecimiento de un voltaje nominal del sistema, se determina la cantidad de módulos que se deben conectar en serie para que el generador fotovoltaico tenga la tensión nominal que se estableció para el sistema —véase ecuación (5)—.

\#Móduloenserie $=\frac{V_{\text {sistema }}}{V_{\text {nominal del módulo }}}$

En este caso de estudio se trabajó con un panel de 250 W con un voltaje de 29,8 voltios en potencia máxima y con un voltaje de 220 voltios del sistema eléctrico del transformador de la ESAVI.

Cantidad de módulos en paralelo. Para establecer la cantidad de ramas de módulos fotovoltaicos que se deben colocar en paralelo, se determinan las siguientes variables:

- $\mathrm{Ci}$, Carga equivalente en corriente: en este apartado se evalúa la corriente en amperio hora (Ah), que debe suministrar el generador ante las exigencias de carga propuestas en la operación del sistema —véase ecuación (6)-. 
$C_{i}=\frac{\text { Energía_total_corregida }}{\text { Voltaje_del_sistema }}[A h]$

- Corriente pico del generador: se necesita saber la corriente entregada por el generador cuando este se expone a la radiación Standard de $1.000 \mathrm{~W} / \mathrm{m}^{2}$ (HSS), dado que esta corriente corresponde al punto donde el generador entrega la máxima potencia —véase ecuación (7)—.

I_pico_del_generador $=\frac{C_{i}}{H S S}[A]$

El término HSS corresponde a las horas de sol estándar presentes en el sitio de la instalación del sistema y que fue obtenido a partir de datos de estaciones meteorológicas para la ciudad de Mariquita en el 2015.

- Cantidad de módulos en paralelo: dado que la corriente pico del generador es la suma de la corriente suministrada por el conjunto de paneles que conforman el generador fotovoltaico, la cantidad de módulos en paralelo se define de la siguiente forma — véase ecuación (8)—:

\#Módulos_en_paralelo $=\frac{I_{-} \text {pico_del_generador }}{I_{m} \_ \text {del__módulo }}$

En este caso de estudio se trabajó con un panel de 250 watt con 8,39 amperios de corriente en potencia máxima.

b) Sistemas fotovoltaicos conectados a la red (SFVCR) o de generación distribuida (GD). Según Hernández-Mora (20I2), en los SFVCR, el generador fotovoltaico se interconecta a la red eléctrica a través de un inversor, que es un equipo que convierte la corriente directa (DC) generada por el arreglo de módulos a corriente alterna (AC). En los SFVCR, la energía es inyectada a la red cuando la generación supera al consumo en un determinado instante y extrae energía de ella en caso contrario.

Desde el punto de vista energético, los SFVCR representan la aplicación de la energía solar fotovoltaica de mayor eficiencia, puesto que la generación tiene lugar en el sitio de consumo (se evitan pérdidas de transporte y distribución) con pocas pérdidas de transformación (los inversores operan típicamente a elevados niveles de eficiencia y en baja tensión) y puede aprovecharse en su totalidad, debido a la elevada fiabilidad de la red. En este estudio se adoptó por un sistema de generación distribuida, puesto que la idea es suprimir las baterías que generan un alto costo al tener que ser sustituidas cada año.

c) Funcionamiento de un SFVCR. La figura 5 muestra esquemáticamente un diagrama de bloques de un SFVCR típico de aplicación en generación distribuida. Los seis bloques funcionales que lo conforman se describen a continuación (Hernández-Mora, 20I2):

- Generador fotovoltaico, formado por los módulos fotovoltaicos y su correspondiente estructura soporte.

- Acondicionador de potencia, responsable de adaptar las características de la energía producida por el generador (DC a tensión variable) a las requeridas por la red eléctrica ( $A C$ a 120 ○ $208 \mathrm{~V}$ ), también llamado convertidor $D C / A C$ o inversor.

- Panel general de servicios o punto de acople común (PCC - point of common coupling), donde se encuentran las protecciones, o conjunto de elementos y medidas adoptadas para garantizar la seguridad del propio SFVCR y la de la red eléctrica.

- Contador bidireccional de energía AC, el cual registra no solo la energía que se consume de la red, sino también la que se podría estar entregando al sistema interconectado en un instante dado.

- Carga, formada por todas aquellas aplicaciones que demandan energía eléctrica para su funcionamiento.

- Red eléctrica convencional. El SFV trabaja de la siguiente forma: la energía eléctrica DC producida por el generador FV se transforma en corriente alterna, con unas características idénticas a la corriente eléctrica de la red, y se interconecta automáticamente con ella. Este proceso lo realiza el inversor, que transforma la corriente continua de los paneles solares a corriente alterna, con una tensión de salida estable y una corriente variable en función de la irradiancia solar. La corriente alterna generada por el inversor se sincroniza con la frecuencia de la corriente de la red $y$, después de pasar por el contador, será inyectada a la red.

La energía no consumida en el mismo sitio de la instalación nunca se pierde, puesto que esta se exporta a la red general de distribución, para que sea consumida por 
Figura 5. Diagrama de bloques de un sistema fotovoltaico interconectado a la red eléctrica

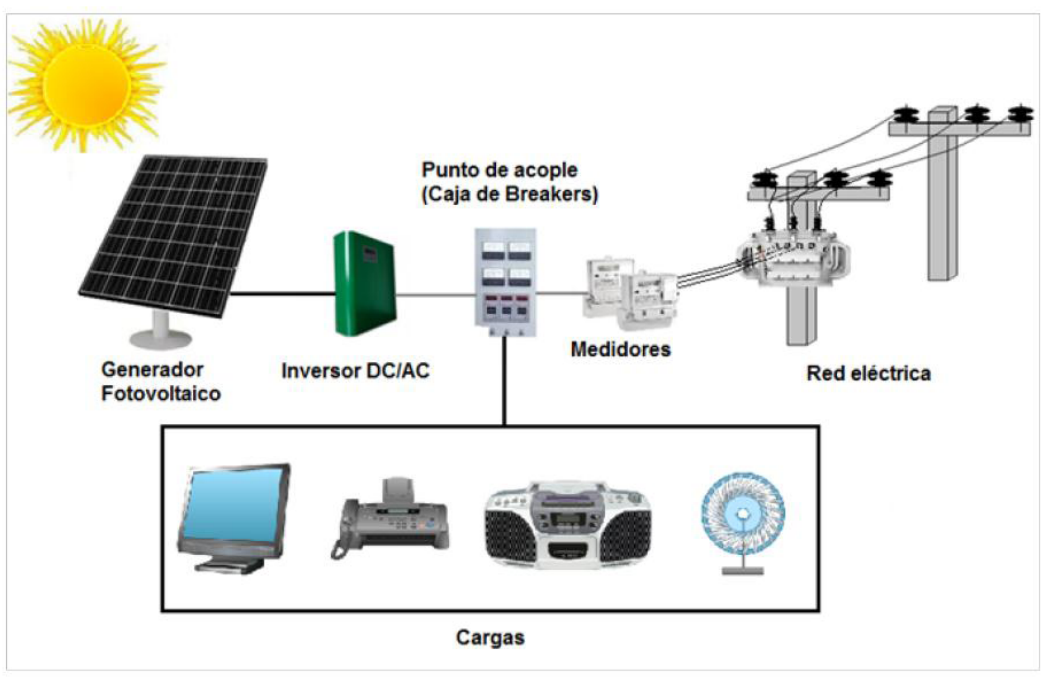

Fuente: Hernández-Mora (2012).

el cliente eléctrico más próximo. Por consiguiente, esta electricidad siempre es consumida a muy corta distancia de donde es producida y, por tanto, las pérdidas asociadas a su transporte son insignificantes.

En este estudio, se espera implementar este tipo de generación, y se desea que lo producido por el sistema fotovoltaico en cerca de cinco horas de disponibilidad solar sea consumido por la ESAVI y cuando no haya suficiente radiación solar la Institución pueda tomar de la red eléctrica la energía requerida. De igual forma, por ejemplo, los fines de semana que no haya consumo apreciable por parte de la ESAVI se pueda exportar energía a la red; lo cual permitirá que el contador bidireccional pueda restar la energía consumida de la red con la producida por el sistema fotovoltaico.

d) Hora solar estándar (HSS) ciudad de Mariquita, año 20I5. En el presente trabajo se pretende analizar el recurso solar de Mariquita, tomando como fuentes de información solo las estaciones meteorológicas presentes en la ciudad que cuenten con instrumentos que midan de manera directa la radiación solar incidente. Se descartan, de esta forma, las estimaciones matemáticas y se parte únicamente de los datos reales obtenidos con las diferentes estaciones. Lo anterior, con el fin de establecer si las condiciones de radiación de Mariquita permitirían el dimensionamiento de sistemas solares fotovoltaicos interconectados residenciales.
La ciudad de Mariquita se encuentra ubicada a $5^{\circ} 12^{\prime} 4^{\prime \prime}$ de latitud norte y $74^{\circ} 54^{\prime} 46^{\prime \prime}$ de longitud occidente. Su temperatura promedio es de $27^{\circ} \mathrm{C}$ (IHOBE.Pública de Gestión Ambiental Bilbao, 2009). Sin embargo, en esta ciudad solo se cuenta con una estación meteorológica en el Aeropuerto José Celestino Mutis, que no mide la radiación solar sino las variables que afecten el despegue, circulación o aterrizaje de los aviones o helicópteros, como la velocidad del viento, presión ambiente, humedad relativa, entre otras. Para obtener entonces la hora solar estándar (HSS), la cual permite realizar comparaciones entre estaciones que manejan unidades de radiación y frecuencias de registro diferentes, se utilizó el software RETScreen (Natural Resources Canada, 20I4); además, dicho valor permitió determinar el área de los paneles solares que se propondrá para la ESAVI sede Mariquita.

En la tabla 2 se observa que la mayor radiación solar en la ciudad de Mariquita se alcanza en la franja horaria de 10 a.m. a 3 p.m. (5 horas), aproximadamente.

De acuerdo con el mapa de disponibilidad horaria de la Unidad de Planeación Minero Energética (UPME), se tenía en el 2005 para la ciudad de Mariquita un valor de HSS mensual de 3,5 a 5 horas (Unidad de Planeación Minero Energética [UPME], 2005) por mes; asimismo, una disponibilidad horaria de 4 a 5 horas de brillo solar (Unidad de Planeación Minero Energética [UPME], 2005). 
Tabla 2. HSS por mes de Mariquita

\begin{tabular}{|c|c|}
\hline Mes & HSS- I.000 W/m \\
\hline Enero & 4,77 \\
\hline Febrero & 5,19 \\
\hline Marzo & 5,36 \\
\hline Abril & 4,51 \\
\hline Mayo & 4,02 \\
\hline Junio & 3,60 \\
\hline Julio & 4,11 \\
\hline Agosto & 3,94 \\
\hline Septiembre & 3,72 \\
\hline Octubre & 3,66 \\
\hline Noviembre & 3,58 \\
\hline Diciembre & 3,91 \\
\hline Promedio anual & 4,19 \\
\hline
\end{tabular}

Fuente: elaboración propia a partir de datos de la estación meteorológica. Software RETScreen. Año 2016.

Por otro lado, se pueden comparar los datos obtenidos en un estudio realizado en la ciudad de Bogotá a ocho estaciones meteorológicas en el 2010, en donde se obtuvo un HSS anual de 4,3I horas (Vallejo-Lozada, Hernández \& Sáenz, 2010) y en la ciudad de Ibagué, Tolima, con 4,88 (Vargas-Galván, 2015), lo cual indica que los valores obtenidos para Mariquita del HSS anual y mensual están dentro de los rangos permitidos, y se puede hacer el cálculo de área y del número de paneles requeridos por la ESAVI.

\section{e) Dimensionamiento del sistema fotovoltaico} para el Área Académica de la ESAVI. A continuación, se muestra el resumen del diseño de los sistemas fotovoltaicos conectados a un punto común de baja tensión, teniendo en cuenta las fórmulas mencionadas ante- riormente. De igual manera, el dimensionamiento se hizo teniendo en cuenta la hora solar estándar por mes dado por el software RETScreen y la UPME.

- Consumo de energía del Área Académica de la ESAVI. En la tabla 3 se observa el resultado de la energía activa corregida; la cual tiene una energía activa de 65,52 kWh por día laboral (30\% del consumo normal del Área Académica). Lo anterior, teniendo en cuenta que aún no se ha reglamentado por la Comisión de Regulación de Energía y Gas (CREG) el uso de créditos energéticos a través de contadores bidireccionales.

Debe tenerse en cuenta que al producir mayor cantidad de energía de la que se requiere y no sea consumida en su totalidad por el Área Académica, esta se perderá; puesto que aún Enertolima no ha reglamentado el uso de contadores bidireccionales para el pago de dichos saldos energéticos, que se producen al restar la energía producida de la red eléctrica con la de los paneles solares del usuario.

Asimismo, se recomienda instalar el sistema fotovoltaico con el contador bidireccional para consumo interno de la Institución;y cuando en el año 2018 la CREG expida la reglamentación de los créditos energéticos, se logrará hacer la sincronización con la red eléctrica de Enertolima y terminar de instalar los paneles solares que generen el resto de energía eléctrica del Área Académica.

De igual forma, en la tabla 4 se encuentra el resumen de los cálculos del sistema fotovoltaico, teniendo en cuenta un panel solar de 260 watt y un inversor de conexión a red trifásica de 15.000 watt, la cual evidencia que se tienen los sistemas fotovoltaicos para el consumo de la ES-

Tabla 3. Energía activa corregida de la ESAVI para el Área Académica.Año 2016

\begin{tabular}{|c|c|c|c|c|c|c|c|}
\hline Año & Mes & $\begin{array}{c}\text { Número de } \\
\text { días }\end{array}$ & $\begin{array}{l}\text { Energía activa } \\
(\mathrm{kWh}) \times \text { mes }\end{array}$ & $\begin{array}{l}\text { HSS- } 1.000 \\
+-100 \mathrm{~W} / \mathrm{m}^{2}\end{array}$ & Energía AC & $\begin{array}{c}\text { Energía activa } \\
(\mathbf{k W h}) \times \text { día }\end{array}$ & $\begin{array}{c}\text { Energía total } \\
\text { corregida en } \\
\text { Wh }\end{array}$ \\
\hline 2015 & Diciembre & 31 & $2.031,12$ & 4,19 & 2.184 & 70,452 & 70.452 \\
\hline
\end{tabular}

Tabla 4. Número de paneles solares y área requerida para el Área Académica de la ESAVI.Año 2016

\begin{tabular}{|c|c|c|c|c|c|c|c|c|}
\hline $\begin{array}{l}\text { Módulos en se- } \\
\text { rie-260 watt IBC } \\
\text { solar }(208 \mathrm{v} / 30,4)\end{array}$ & $\begin{array}{l}\text { Carga equiva- } \\
\text { lente en corri- } \\
\text { ente (Ah) }\end{array}$ & $\begin{array}{l}\text { Cor- } \\
\text { riente } \\
\text { pico del } \\
\text { genera- } \\
\text { dor (A) }\end{array}$ & $\begin{array}{l}\text { Módulos en } \\
\text { paralelo-260 } \\
\text { watt IBC } \\
\text { solar (8.23 } \\
\text { A) }\end{array}$ & $\begin{array}{l}\text { Cantidad } \\
\text { de paneles }\end{array}$ & $\begin{array}{l}\text { Can- } \\
\text { tidad } \\
\text { real de } \\
\text { paneles }\end{array}$ & $\begin{array}{l}\text { Área } \\
\left(m^{2}\right)\end{array}$ & $\begin{array}{l}\text { Inversor por } \\
\text { agrupación } \\
\text { de } 64 \text { pan- } \\
\text { eles solares } \\
\text { de } 260 \text { watt- } \\
\text { I5 kVA }\end{array}$ & $\begin{array}{l}\text { Potencia paneles - } \\
\text { Teniendo en cuenta } \\
\text { el HSS y } 260 \text { watt y } \\
\text { eficiencia del } 97 \%- \\
\text { Wh }\end{array}$ \\
\hline 6 & 320 & 76 & 10 & 60 & 60 & 111 & I & 63.403 \\
\hline
\end{tabular}


Figura 6. Número de paneles solares por inversor de 15.000 watt

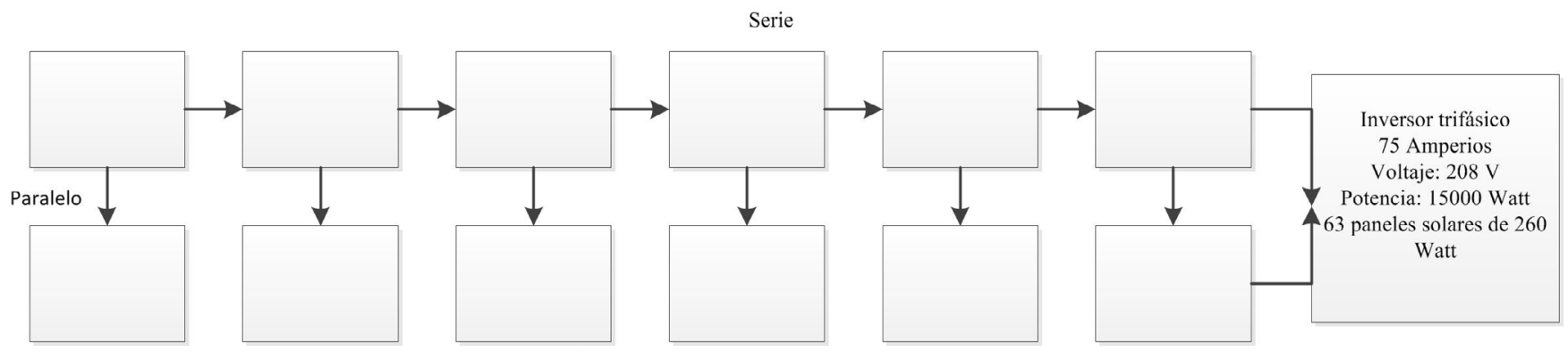

AVI y su respectivo arreglo de paneles. De igual modo, se propone un arreglo de 63 paneles solares para aumentar la energía producida (2.03l kWh).

Por otro lado, el número de paneles solares por inversor se puede ver en la figura 6; donde las filas son paneles en serie y las columnas paneles en paralelo.

\section{f) Fichas técnicas de los equipos seleccionados}

- Panel solar de 260 watt policristalino, distribuido por la empresa IBC Solar (IBC Solar, 2016).

- Inversor trifásico Fronius 15.0-3 para conexión a red con una capacidad de 15.000 watt, distribuido por la empresa Hybrytec (Hybrytec, 2016). El cual garantiza el monitoreo de producción de energía a través de redes de Internet WiFi.

- Contador bidireccional EA/R 5-IOA CL 02s 480V C/ OP Modem referencia SL 7000, distribuido por la empresa Itrón con sucursal de distribución en Colombia (Itrón, 2015).

g) Montaje de la estructura, ángulo azimut o de inclinación y declinación magnética (norte verdadero). Para el montaje de los 63 paneles solares, se decide hacerlo sobre el parqueadero de motos cerca al Área Académica (lado derecho) como se muestra en la figura 7 , el cual cuenta con unas dimensiones de $30,6 \mathrm{~m}$ de largo $x$ 6,23 m de ancho con perlines en $C$, teja (cubiertos) y un ángulo de inclinación de aproximadamente $2^{\circ}$; lo anterior, con la finalidad de reutilizar la estructura metálica que se tiene en la actualidad para su montaje y no incurrir en sobrecostos por obras civiles adicionales.
Figura 7. Montaje propuesto para los paneles solares del Área Académica. Año 2016

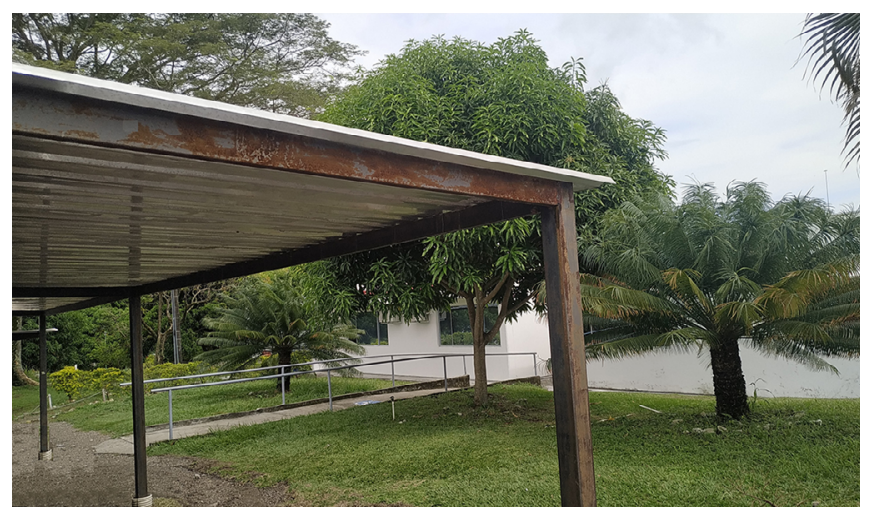

De igual modo, se recomienda un montaje de los paneles solares como se puede ver en la figura 8 , con ángulos o perfiles en $C$ en aluminio que van apoyados sobre los perlines de la estructura metálica actual, con un ángulo de inclinación vertical de aproximadamente $14^{\circ}$ y con una inclinación horizontal de $2^{\circ}$ hacia el norte verdadero; esto, teniendo en cuenta la latitud de Mariquita, Tolima, y la declinación magnética para el planeta tierra.

Figura 8. Estructura soporte de los paneles solares para el Área Académica.Año 2016.

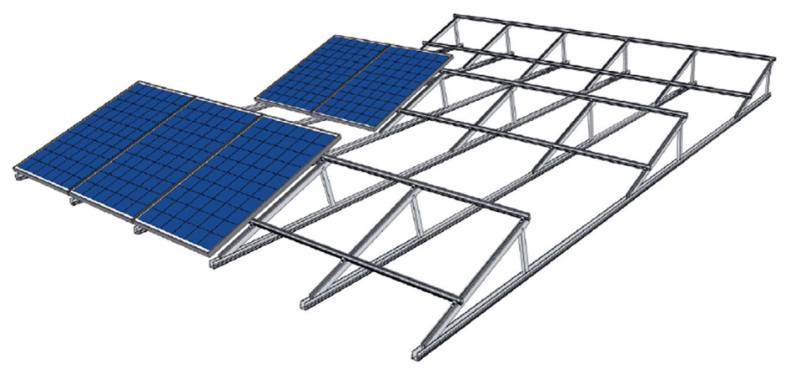

Fuente: elaboración propia. 


\section{Cálculo de emisiones de dióxido de carbono equivalente $\left(\mathrm{CO}_{2 \mathrm{eq}}\right)$}

Las emisiones indirectas de gases de efecto invernadero (GEI) por energía, corresponden a las de $\mathrm{CO}_{2 \text { eq }}$ que provienen de la generación de la energía eléctrica comprada por la ESAVI a Enertolima. Según la Unidad de Planeación Minero Energética-UPME (2015), el factor mix de electricidad promedio mensual para el 2015 en Colombia fue de $0,21 \mathrm{tCO}_{2 \mathrm{eq}} / \mathrm{MWh}$, lo cual indica que de acuerdo con el sistema fotovoltaico propuesto con una generación de energía eléctrica de $1,902 \mathrm{MWh} /$ mes se puede estimar dejar de emitir 0,39 $\mathrm{tCO}_{2 \mathrm{eq}} /$ mes al medioambiente.

\section{Análisis económico de la propuesta del sistema fotovoltaico}

A continuación, se realiza el análisis económico de la propuesta para el Área Académica de la Escuela de Aviación Policial.

\section{Presupuesto}

El presupuesto de la implementación de paneles solares para satisfacer el consumo energético del Área Académica de la Escuela de Aviación Policial fue de \$ 94.980.850; el cual incluye servicio técnico para la instalación eléctrica y montaje de la estructura, materiales e insumos y los equipos que conforman el sistema fotovoltaico como paneles solares, inversor y el contador bidireccional.

Valor actual neto (VAN) y tasa interna de retorno (TIR)

Se determinó que el tiempo de recuperación de la inversión era de aproximadamente II años con un valor de $\$$ II.908.342 y la TIR para el 204I era de 10,70\% para la implementación de paneles solares en el Área Académica de la ESAVI; además, se tuvo en cuenta que la vida útil de los paneles solares es de cerca de 25 años y una tasa de usura del $0 \%$. De igual modo, se tuvo en cuenta la depreciación para maquinaria y equipos.

\section{Análisis y discusión de resultados}

\section{Análisis de consumos energéticos por hora y día del Área Académica de la ESAVI}

En el análisis por hora y día se logró identificar que en horas no laborales (después de las 10 p.m. hasta las 6 a.m. del otro día) o al mediodía se presentan consumos de energía activa promedio de $9,16 \mathrm{kWh}$ y reactiva de $1,7 \mathrm{kVArh}$, los cuales son bastante elevados teniendo en cuenta que no hay personas dentro de las aulas académicas $u$ oficinas; además, indican que posiblemente en estos horarios se dejan equipos encendidos como aires acondicionados, luminarias, equipos de cómputo, entre otros. De igual forma, en los fines de semana (sábado y domingo), también existe un consumo promedio de energía activa de 6,96 $\mathrm{kWh}$ y reactiva de 0,93 kVArh, y que puede ser causado por la misma situación.

Se recomienda hacer un estudio de gestión energética que incluya un análisis de calidad de energía y termografía, que permita elaborar propuestas a corto, mediano y largo plazo, como:

- Implementación de sensores de presencia para el encendido de las luminarias de los pasillos, oficinas o aulas académicas.

- Cambio de iluminación fluorescente o incandescente a tecnología LED.

- En el caso de los aires acondicionados: uso de desincrustante, cambio de refrigerante, $y$ recambio por equipos de alta eficiencia o bioclimatizadores.

- Banco de condensadores para la reutilización de la energía reactiva.

- Sistemas inteligentes de medición de energía eléctrica que apaguen los equipos cuando no hayan personas en las aulas académicas $u$ oficinas.

\section{Capacidad del sistema fotovoltaico con conexión a red}

Se llevó a cabo un dimensionamiento de un arreglo de paneles solares con conexión a red; el cual cuenta con 63 paneles solares de 260 watt, un inversor trifásico de 15.000 watt, un contador bidireccional, una estructura en aluminio, entre otros elementos. Además, se espera que pueda generar 16,38 kWp/hora y que sea instalado en el parqueadero de motos del lado derecho del Área Académica.

Emisiones de dióxido de carbono equivalente $\left(\mathrm{CO}_{2 \mathrm{eq}}\right)$ no emitidas al medioambiente

Se espera dejar de emitir aproximadamente $0,39 \mathrm{tCO}_{2 \mathrm{eq}} /$ mes al medioambiente con la implementación del sistema fotovoltaico diseñado en la ESAVI, lo cual contribuye a mejorar el factor económico por el ahorro de energía 
eléctrica y la mitigación del calentamiento global y, por ende, del cambio climático. De igual forma, se concluye que los sistemas fotovoltaicos son energías renovables y limpias, puesto que no agotan los recursos naturales, evitan la contaminación del medioambiente por combustibles fósiles (energías convencionales) y cuentan con una fuente de energía ilimitada como la solar, permitiendo la sostenibilidad ambiental del proyecto y la posibilidad de generación de una conciencia y cultura ambiental en los funcionarios y estudiantes de la Escuela, los cuales pueden servir como multiplicadores de dicha importancia en su entorno familiar, social y laboral.

\section{Conclusiones}

Con el análisis del consumo energético del Área Académica de la Escuela de Aviación Policial, objeto de estudio, se logró determinar registros elevados en consumos de energía activa y reactiva en horas de la noche ( 10 p.m. a 6 a.m. del siguiente día), a mediodía o el fin de semana donde no se realizan actividades académicas y laborales por parte del personal del Área (funcionarios y estudiantes), los cuales pueden ser generados por dejar encendidos equipos como aires acondicionados y cómputo; iluminación de oficinas, aulas académicas y pasillos, entre otros. De igual forma, las áreas con los respectivos consumos energéticos promedio $(\mathrm{kWh})$ más elevados en ese horario fueron las oficinas con $10.18 \mathrm{I}$, la sala de idiomas con 3.446; los simuladores con 3.050 y la biblioteca con 2.735. Además, se determinó que se tienen problemas en los circuitos eléctricos como fluctuaciones constantes de voltaje, altos valores de energía reactiva, fases de carga desniveladas, entre otros, y se recomienda hacer un análisis de calidad de energía que incluya preferiblemente la termografía.

El sistema fotovoltaico con conexión a red propuesto para la ESAVI es viable desde el punto de vista financiero y puede ser implementado, puesto que se espera recuperar la inversión en aproximadamente II años (año 2027) a partir de implementar el sistema fotovoltaico propuesto en el 2016. Además, se determina su sostenibilidad ambiental con el ahorro de energía eléctrica que se deja de producir de fuentes convencionales como la hidráulica 0 combustibles fósiles y las emisiones de $0,39 \mathrm{tCO}_{2 \mathrm{eq}} / \mathrm{mes}$ que se dejan de emitir al medioambiente, contribuyendo con ello a la mitigación del calentamiento global y, por ende, del cambio climático.
La implementación de los sistemas fotovoltaicos con conexión a red son importantes en este tipo de instituciones académicas de la Policía Nacional, puesto que permite las condiciones de confort de los estudiantes y funcionarios al no generar sobrecostos por consumo de energía eléctrica cuando llevan a cabo a diario sus funciones, y se espera con ello una mayor eficiencia y eficacia en sus actividades, sobre todo en épocas de verano que pueden alcanzar temperaturas ambiente entre $25^{\circ} \mathrm{C}$ y $32{ }^{\circ} \mathrm{C}$ en algunos departamentos de Colombia (Instituto de Hidrología, Meteorología y Estudios Ambientales-IDEAM, 20I5). También, si se logra implementar este tipo de sistemas con una generación parcial o completa de energía eléctrica en las demás escuelas o compañías de la Policía Nacional en el territorio nacional, los resultados contribuirían a incrementar la matriz energética del país, el cumplimiento de algunos de los objetivos de desarrollo sostenible para Colombia, promover e incentivar la implementación de energías limpias o renovables en el país, y a la generación de una conciencia y cultura ambiental en los funcionarios y estudiantes de la Institución, los cuales sirvan como multiplicadores del cuidado del medioambiente en su entorno familiar, social y laboral.

\section{Referencias}

Campos-Avella, J. C., Gómez-Dorta, R., \& Santos-Macías, L. (1997). La eficiencia energética en la gestión empresarial. Cienfuegos, Cuba: Universidad de Cien Fuegos.

Carneiro, J. (2009). Dimensionamento de sistemas fotovoltaicos. Recuperado el $1^{\circ}$ de mayo de 2015 , de http://repositorium.sdum.uminho.pt/handle//822//6965.

Domínguez-González, H. (20I2). Diseño de un sistema fotovoltaico para la generación de energía eléctrica en el COBAEV 35 XALAPA (tesis de Maestría). Xalapa-Enríquez: Universidad Veracruzana.

Hernández-Mora, A. J. (2012). Metodología para el análisis técnico de la masificación de los sistemas fotovoltaicos como opción de generación distribuida en redes de baja tensión (Tesis doctoral). Bogotá: Universidad Nacional de Colombia.

Hybrytec. (2016). Catálogo de inversores trifásicos. Recuperado el 17 de enero de 2016, de http://www.hybrytec.com/.

IBC Solar. (2016). Catálogo de paneles solares. Recuperado el 17 de enero de 2016, de https://www.ibc-solar.es/.

IHOBE.Pública de Gestión Ambiental Bilbao. (19 de noviembre de 2009). IHOBE. Recuperado de http://www.ihobe. 
net/Publicaciones/Ficha.aspx?IdMenu=750e07f4- I I a440da-840c-0590b9lbc032\&Cod = bc53a7db-3edb-4b96ac9a-Ifl63ed0d76b.

Instituto de Hidrología, Meteorología y Estudios Ambientales-IDEAM. (20I5). Anuario climatológico. Recuperado de http://www.ideam.gov.co/web/tiempo-y-clima/climato logico-mensual/-/document_library_display/xYvIPc4uxk I Y/view/299660?_II0_INSTANCE_xYvIPc4uxkIY_redirect=http\%3A\%2F\%2Fwww.ideam. gov.co\%2Fweb\%2Ftiempo-y-clima\%2Fclimatologicomensual\%3Fp_P_id\%3DII0_INSTANCE_x.

Islam, M., Mekhilef, S., \& Hasan, M. (20I5). Single phase transformerless inverter topologies for grid-tied photovoltaic system: A review. Renewable and Sustainable Energy Reviews, (45), 69-86.

Itrón.(20I5). Catálogo de contadores bidireccionales. Recuperado de https://www.itron.com/mxca/es/pages/default.aspx?re gion=latinAmerica\&language $=$ spanish .

Natural Resources Canada. (I0 de octubre de 20I4). RETScreen International. Recuperado de 2015, de http://www. retscreen.net/es/home.php.

Norma Técnica Colombiana (NTC). (10 de junio de 2008). Norma NTC 500I. Recuperado de http://tienda.icontec. org/brief/NTC500I.pdf.

Patiño-Abella, J. S., Tello-Reyes, J. S., \& Hernández-Mora, J. A. (20l3). Diseño e implementación de un sistema fotovoltaico híbrido y desarrollo de su regulador de carga aplicando instrumentación virtual. Elementos , 2(I), 29-45.
Tsang, K., \& Chan, W. (20/4). Rapid islanding detection using multi-level inverter for grid-interactive PV system. Energy Conversion and Management, (77), 278-286.

Unidad de Planeación Minero Energética (UPME). (2005). Mapas de brillo solar. Recuperado el I5 de enero de 2015, de http://www.upme.gov.co/Docs/Atlas_Radiacion_Solar/3Mapas_Brillo_Solar.pdf.

Unidad de Planeación Minero Energética (UPME). (2005). Mapas de radiación solar global sobre una superficie plana. Recuperado el 15 de enero de 2015, de http://www. upme.gov.co/Docs/Atlas_Radiacion_Solar/2-Mapas_Radiacion_Solar.pdf.

Unidad de Planeación Minero Energética-UPME. (20I5). Factor mix eléctrico de la UPME para Colombia año 20I5. Bogotá: UPME.

Vallejo-Lozada,W.A., Hernández, J., \& Sáenz, E. (2010). Estudio del recurso solar en la ciudad de Bogotá para el diseño de sistemas fotovoltaicos interconectados residenciales. Revista Colombiana de Física, 42(2), 5.

Vargas-Galván, G. A. (20I5). Programa piloto para la gestión energética en instituciones educativas en el departamento del Tolima (Tesis de maestría). Ibagué: Universidad de Ibagué. 